\title{
A CFH12K Survey of Red Giant Stars in the M81 Group
}

\author{
Patrick R. Durrell, Megan E. DeCesar, Robin Ciardullo \\ Department of Astronomy $\&$ Astrophysics, Penn State University, \\ University Park, PA USA \\ Denise Hurley-Keller, John J. Feldmeier \\ Department of Astronomy, Case Western Reserve University, \\ Cleveland, OH, USA
}

\begin{abstract}
.
We present the preliminary results of a wide-field photometric survey of individual red giant branch (RGB) and asymptotic giant branch (AGB) stars in the M81 group, performed with the CFH12K mosaic camera of the CFHT. We use deep VI images of $0.65 \mathrm{sq}$. deg. of sky to map out the two-dimensional distribution of intragroup stars and to search for stars associated with the many $\mathrm{HI}$ tidal tails in the group. We place an upper limit on the presence of metal-poor RGB stars in a field located 50-80 kpc from M81, and derive an 'intragroup' fraction of $<2 \%$. In a field sampling the M81-NGC3077 HI tidal tail, we find blue stars associated with some of the tidal features, including 2 clumps which we tentatively describe as tidal dwarf candidates. These objects are $\sim 1 \mathrm{kpc}$ in size, and, based on their color-magnitude diagrams, have formed stars as recently as $\sim 30-70$ Myr ago, long after the group's most recent interactions.
\end{abstract}

Tidal encounters often liberate stars from their parent galaxies (e.g., Moore et al. 1996). Intracluster red giants and planetary nebulae are excellent tracers of such stars and have been observed in the cluster environments of Virgo and Fornax (Ferguson et al. 1998; Durrell et al. 2002; Feldmeier et al. 2003; Arnaboldi et al. 2003), where high-speed encounters are important. However, little is known about their presence in smaller groups: do 'intragroup' stars exist? The M81 system is the nearest group of galaxies to have undergone recent interactions $(\sim 220-280$ Myr ago; Yun 1999), and the group is filled with tidally-produced HI (Yun et al. 1994). As such, it provides an excellent laboratory to search for stars extracted from previous encounters.

We have performed a deep VI imaging survey (using the CFH12K camera at the CFHT; field size 0.33 sq. degrees) to resolve the brightest stars in the M81 group. Two group fields have been imaged, with a third field (located $10^{\circ}$ away) taken to quantify the sizeable contamination from background galaxies. Typical limiting magnitudes of our survey are $V \sim 25.5, I \sim 24.5$.

Since our Field 2 data (located $50-80 \mathrm{kpc}$ from M81) are only just deep enough to reach the RGB tip, we can only place a rough upper limit on the presence of intragroup stars. Using the $V I$ color magnitude diagram, number counts for all unresolved objects in the 5 deepest chips of the CFH12K array, 
and a statistical subtraction of a background CMD, we find an excess of $1.0 \pm 0.4$ RGB stars $\operatorname{arcmin}^{-1}$. This extrapolates to a surface brightness of $\mu_{V} \sim 31$. If this luminosity density is constant over the $\sim 7$ sq. deg. of the group's core, then the total intragroup $V$-band luminosity is $<6 \times 10^{8} L_{\odot}$, or $<2 \%$ of the group's total luminosity. Note that this estimate does not include the likely presence of M81 halo stars nor the contribution of a metal-rich population.
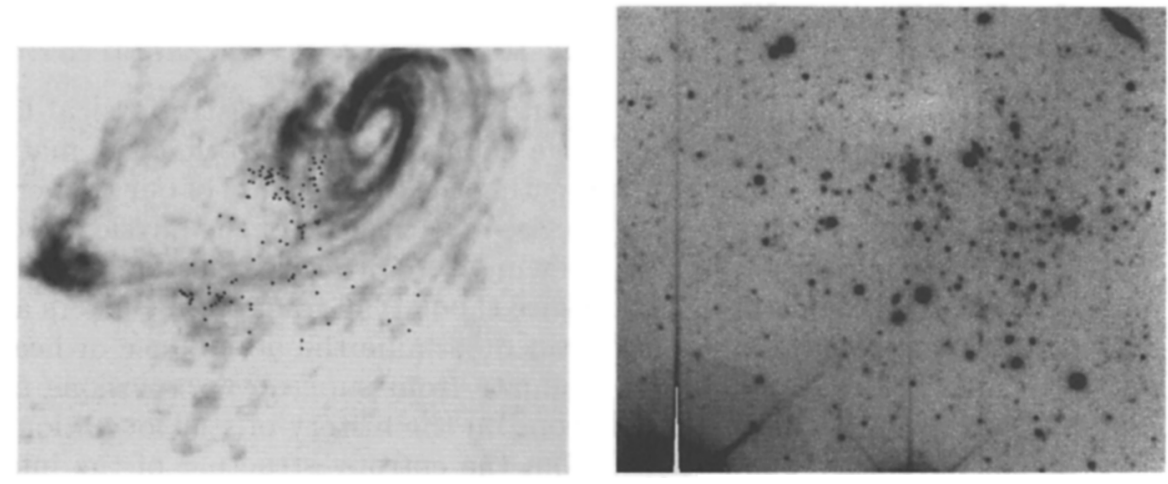

Figure 1. Left: Locations of blue stars in our Field 1 CMD, superimposed on the HI map of Yun et al. (1994). Contamination from foreground stars and background galaxies is $\sim 20 \%$. Right: $V$ image of the region in the HI South Tidal Arm that contains the blue star clump.

Our data also indicate the presence of blue $(V-I<0.4)$ stars associated with some of the group's HI tidal streams. As shown in Fig. 1, there is a clump of blue objects located in the densest HI feature of the Southern Tidal Arm. This feature, which is considered an 'HI satellite' by Yun et al. (1994), is about $\sim 1^{\prime}$ ( 1 $\mathrm{kpc}$ ) across. Isochrones matched to the object's CMD, and that of an adjacent clump, suggest that star formation occurred $30-70 \mathrm{Myr}$ ago, well after the tidal encounters which created the $\mathrm{HI}$ arm. These star-forming clumps are tidal dwarf candidates.

Acknowledgments. We would like to thank Chris Mihos and George Jacoby for related discussions, and Min Yun for providing us with his HI map.

\section{References}

Arnaboldi, M., et al. 2003, AJ, 125, 514

Durrell, P.R., et al. 2002, ApJ, 570, 119

Feldmeier, J.J., Ciardullo, R., Jacoby, G.H., \& Durrell, P. 2003, ApJS, 145, 65

Ferguson, H.C., Tanvir, N.R., \& von Hippel, T. 1998, Nature, 391, 461

Moore, B., et al. 1996, Nature, 379, 613

Yun, M.S., Ho, P.T.P., \& Lo, K. Y. 1994, Nature, 372, 530

Yun, M.S. 1999, in IAU Symposium 186: Galaxy Interactions at Low and High Redshift, ed. J.E. Barnes \& D.B. Sanders, p. 81 